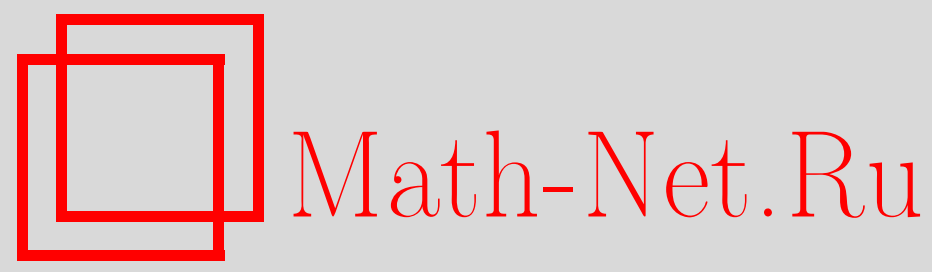

А. М. Магомедов, K вопросу о реберной раскраске двудольного графа, Дискрет. матем., 2009, том 21, выпуск 2, 153158

DOI: https://doi.org/10.4213/dm1055

Использование Общероссийского математического портала Math-Net.Ru подразумевает, что вы прочитали и согласны с пользовательским соглашением http://www . mathnet.ru/rus/agreement

Параметры загрузки:

IP : 34.229 .45 .116

26 апреля 2023 г., 17:43:52

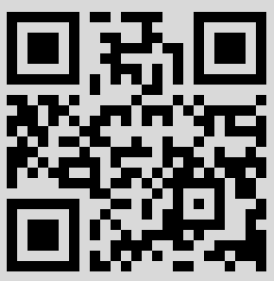




\title{
К вопросу о реберной раскраске двудольного графа
}

\author{
(ㄷ) 2009 г. А. М. Магомедов
}

\begin{abstract}
Для двудольных графов $G(X, Y, E)$, в которых степени вершин $y \in Y$ не превосходят $q$, а степени вершин $x \in X$ равны $2 q$, рассматривается задача существования реберной 2-раскраски такой, что любой вершине $x \in X$ инцидентны $q$ ребер каждого цвета, а цвета ребер, инцидентных вершине $y \in Y$, одинаковы.
\end{abstract}

\section{1. Определения и обозначения}

Класс двудольных графов $G(X, Y, E)$, в котором степени вершин $x \in X$ равны $2 q, q \in \mathbf{Z}^{+}$, а степени вершин $y \in Y$ не превосходят $q$ (равны $q$ ), обозначим через $C_{q}$ (соответственно, через $\left.C_{q}^{0}\right)$. Будем обозначать 2-раскраску ребер графа $G(X, Y, E)$ из класса $C_{q}$ в цвета +1 и -1 разбивающей, если среди ребер, инцидентных вершине $x \in X$, имеется по $q$ ребер каждого цвета, а цвета всех инцидентных вершине $y \in Y$ ребер равны одному и тому же значению $c(y)$. При этом $c(y)$ будем называть цветом вершины $y$, а цвет ребра $(x, y)$ будем обозначать через $c(x, y)$. Будем говорить, что цвет $i$ представлен $k$ раз в раскраске в вершине $u$, если в точности $k$ ребрам, инцидентным вершине $u$, присвоен цвет $i$. Граф будем называть раскрашиваемым или нераскрашиваемым в зависимости от существования разбивающей раскраски.

Теорема 1. Любой граф класса $C_{2}$ является раскрашиваемым.

Доказательство. Заметим, что число висячих вершин в графе $G(X, Y, E) \in C_{2}$ четно. Пусть $y_{1}^{\prime}, y_{1}^{\prime \prime}, \ldots, y_{k}^{\prime}, y_{k}^{\prime \prime}-$ множество таких вершин. Для каждого $i, 1 \leqslant i \leqslant k$, выполним следующие действия:

(1) обозначим $e_{i}^{\prime}=\left(v_{i}^{\prime}, y_{i}^{\prime}\right)$ и $e_{i}^{\prime \prime}=\left(v_{i}^{\prime \prime}, y_{i}^{\prime \prime}\right)$ ребра, инцидентные вершинам $y_{i}^{\prime}$ и $y_{i}^{\prime \prime}$ соответственно;

(2) введем новую вершину $y_{i}$ и заменим набор $e_{i}^{\prime}, y_{i}^{\prime}, y_{i}^{\prime \prime}, e_{i}^{\prime \prime}$ на набор, состоящий из вершины $y_{i}$ и двух инцидентных ей ребер $\left(v_{i}^{\prime}, y_{i}\right), y_{i},\left(y_{i}, v_{i}^{\prime \prime}\right)$.

В каждой связной компоненте полученного графа $G^{\prime}\left(X, Y^{\prime}, E\right) \in C_{2}^{0}$ рассмотрим ориентированный эйлеров цикл. В качестве начальной дуги выберем произвольную дугу $\left(y_{0}, x\right)$ цикла с началом в вершине $y_{0} \in Y^{\prime}$ и положим $c\left(y_{0}, x\right)=-1$. Присвоим цвета последовательным дугам цикла $\ldots,\left(v_{i}, v_{j}\right),\left(v_{j}, v_{k}\right), \ldots$, следуя правилу

$$
c\left(v_{j}, v_{k}\right)= \begin{cases}c\left(v_{i}, v_{j}\right), & \text { если } v_{j} \in Y^{\prime}, \\ -c\left(v_{i}, v_{j}\right), & \text { если } v_{j} \in X .\end{cases}
$$


В результате такой раскраски в любой вершине $x \in X$ каждый из цветов представлен два раза, а цвета ребер, инцидентных произвольной вершине $y \in Y, y \neq y_{0}$, одинаковы. Если пронумеровать дуги цикла в порядке их обхода цифрами $0,1,2,3,4, \ldots$, цвета дуг с номерами, кратными четырем, равны -1 . Поскольку число ребер равно $4|X|$ и процесс присвоения цветов завершается в дуге, инцидентной вершине $y_{0}$, то цвета ребер, инцидентных вершине $y_{0}$, также одинаковы. Следовательно, полученная 2-раскраска является разбивающей. Теорема доказана.

Всюду в дальнейшем предполагается, что $L$ и $n$ - целые положительные числа и выбран алфавит $A=\{1,2, \ldots, n\}$.

Следствие 1. Пусть $W$ - набор двухбуквенных слов над алфавитом $A$. Если число вхождений любой буквы из $A$ в слова набора $W$ не превосходит 4 , то $W$ допускает разбиение на два поднабора, в каждом из которых число вхождений любой буквы из $А$ не превосходum 2.

Определение 1. Дефрагментацией матрицы называется преобразование ее к такому виду, в котором наборы элементов в каждой строке и в каждом столбце матрицы сохранены, при этом в каждой строке ненулевые элементы размещены рядом, в последовательных ячейках.

Следствие 2. Если $L \geqslant n$ и каждый столбец, $L \times 4$ матрищы $M$ содержит $L-n$ нулей и все буквы алфавита $A$, а число ненулевых элементов в каждой строке равно двум, то матрица $M$ допускает дефрагментаичию.

Как известно, в некоторых случаях свойство планарности может упростить исследование вопросов раскрашиваемости графа [1].

Теорема 2. Простой граф $G(X, Y, E)$ класса $C_{3}^{0}$ непланарен.

Доказательство. Обозначим $\varphi_{k}$ число $k$-угольных граней в графе $G(X, Y, E) \in C_{3}^{0}$. В графе отсутствуют кратные ребра и грани с нечетным числом вершин, поэтому

$$
\varphi_{2}=0, \quad \varphi_{2 i-1}=0, \quad i=1,2, \ldots
$$

Предположим, что $G$ является планарным графом. Если $v, e$ и $p-$ соответственно число вершин, ребер и граней в $G$, то по формуле Эйлера

$$
v-e+p=2 \text {. }
$$

Перепишем тождество $p=\sum_{i=1,2, \ldots} \varphi_{i}$ с учетом (1) в виде

$$
p=\sum_{i=2,3, \ldots} \varphi_{2 i}
$$

Так как каждое ребро планарного графа входит ровно в две грани, $2 e=\sum_{i=1,2, \ldots} i \varphi_{i}$, откуда, согласно (1),

$$
e=\sum_{i=2,3, \ldots} i \varphi_{2 i}
$$

Поскольку $G(X, Y, E) \in C_{3}^{0}$, справедливо равенство

$$
6|X|=3|Y|=e .
$$




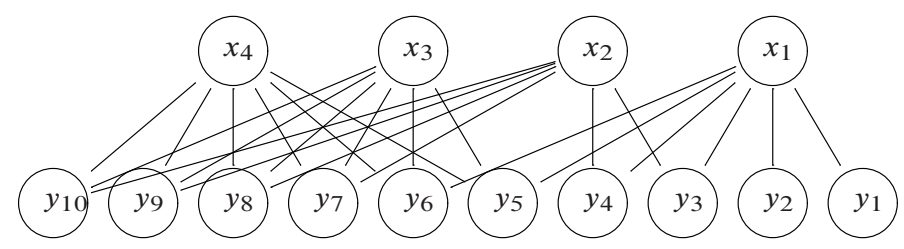

Рис. 1. При любой разбивающей раскраске $c\left(y_{1}\right)=-c\left(y_{2}\right), c\left(y_{3}\right)=-c\left(y_{4}\right)$

Отсюда и из тождества $v=|X|+|Y|$ следует, что $v-e=-e / 2$, или в соответствии с (4)

$$
v-e=-\frac{1}{2} \sum_{i=2,3, \ldots} i \varphi_{2 i} .
$$

Подставляя правые части (3) и (5) в (2), получим равенство

$$
\sum_{i=3,4, \ldots}\left(1-\frac{i}{2}\right) \varphi_{2 i}=2,
$$

где все слагаемые в левой части неположительны. Таким образом, предположение о планарности графа $G$ привело к противоречию. Теорема доказана.

Легко видеть, что не всякий граф класса $C_{3}^{0}$ является раскрашиваемым. Например, нераскрашиваем следующий двудольный мультиграф $G(X, Y, E)$, заданный списками смежных вершин: $y_{1}\left(x_{1}, x_{1}, x_{1}\right), y_{2}\left(x_{1}, x_{2}, x_{2}\right), y_{3}\left(x_{1}, x_{2}, x_{2}\right), y_{4}\left(x_{1}, x_{2}, x_{2}\right)$.

В самом деле, пусть граф $G$ раскрашиваем и выбрана некоторая разбивающая раскраска. Тогда для каждой из трех пар вершин $\left(y_{i}, y_{j}\right)$ множества $\left(y_{2}, y_{3}, y_{4}\right)$ выполняется неравенство $c\left(y_{i}\right) \neq c\left(y_{j}\right)$; в противном случае четыре ребра $\left(y_{i}, x_{2}\right),\left(y_{i}, x_{2}\right),\left(y_{j}, x_{2}\right)$, $\left(y_{j}, x_{2}\right)$ из шести, инцидентных вершине $x_{2}$, были бы одинакового цвета, что противоречит определению разбивающей раскраски. Но три значения $c\left(y_{2}\right), c\left(y_{3}\right)$ и $c\left(y_{4}\right)$, принадлежащие двухэлементному множеству $\{+1,-1\}$, не могут быть попарно различными. Следовательно, рассмотренный граф нераскрашиваем.

Приведенное обоснование нераскрашиваемости опирается на наличие кратных ребер. В приложениях же задача о раскрашиваемости нередко возникает для простых графов. Так, если в следствии 2 теоремы 1 матрица $M$ представляет расписание учебных занятий, требование бесповторности ненулевых элементов в строке является естественным. Поэтому вопрос существования простых нераскрашиваемых графов класса $C_{3}^{0}$ представляет самостоятельный интерес. Выяснить существование подобных графов было предложено О. Б. Лупановым.

Лемма 1. При любой разбивающей раскраске графа $G_{0}$, изображенного на рис. 1 ,

$$
c\left(y_{1}\right)=-c\left(y_{2}\right), \quad c\left(y_{3}\right)=-c\left(y_{4}\right) \text {. }
$$

При любой разбивающей раскраске графа $G^{0}$, изображенного на рис. 2, выполняется хотя бы одно из равенств

$$
c\left(a_{1}\right)=c\left(a_{2}\right), \quad c\left(b_{1}\right)=c\left(b_{2}\right) .
$$




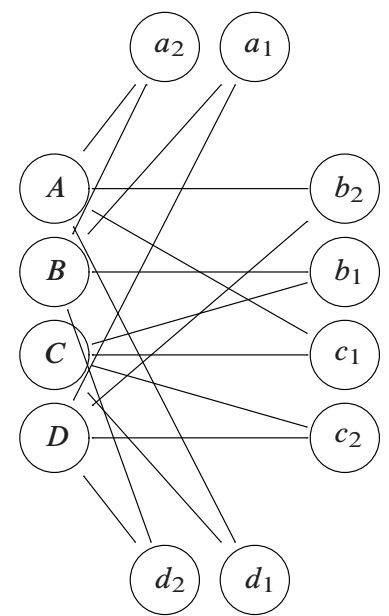

Рис. 2. Граф $G^{0}$. При любой разбивающей раскраске $c\left(a_{1}\right)=c\left(a_{2}\right)$ или $c\left(b_{1}\right)=c\left(b_{2}\right)$

Доказательство. Докажем первое утверждение. Пусть задана разбивающая раскраска графа $G_{0}$ и, для определенности, $c\left(y_{3}\right)=+1$. Рассмотрим последствия равенства $c\left(y_{4}\right)$ значениям +1 и -1 соответственно. Если $c\left(y_{4}\right)=+1\left(c\left(y_{4}\right)=-1\right)$, то $c\left(x_{2}, y_{4}\right)=+1$ $\left(c\left(x_{2}, y_{4}\right)=-1\right)$, и среди цветов четырех ребер $c\left(x_{2}, y_{7}\right), c\left(x_{2}, y_{8}\right), c\left(x_{2}, y_{9}\right), c\left(x_{2}, y_{10}\right)$, инцидентных вершине $x_{2}$ наряду с ребрами $\left(x_{2}, y_{3}\right)$ и $\left(x_{2}, y_{4}\right)$, окрашенными в цвет +1 (в цвета +1 и -1 соответственно), соотношение цветов +1 и -1 составляет $1: 3$ (2:2). Так как вершины $y_{7}, y_{8}, y_{9}, y_{10}$, концевые для этих ребер, входят и в список вершин, смежных $x_{3}$, соотношение цветов +1 и -1 для вершин $y_{5}$ и $y_{6}$, также входящих в этот список, равно $2: 0(1: 1)$.

Таким образом, из условия $c\left(y_{3}\right)=c\left(y_{4}\right)=+1$ следует равенство цветов четырех вершин $y_{3}, y_{4}, y_{5}, y_{6}$, смежных вершине $x_{1}$, что противоречит определению разбивающей раскраски графа класса $C_{3}$. При $c\left(y_{3}\right)=+1, c\left(y_{4}\right)=-1$ вершины $y_{5}$ и $y_{6}$ имеют цвета разных знаков, а поскольку каждый из цветов +1 и -1 представлен в вершине $x_{1}$ три раза, разные знаки имеют тогда и цвета вершин $y_{1}$ и $y_{2}$, смежные вершине $x_{1}$ наряду с вершинами $y_{3}, y_{4}, y_{5}, y_{6}$. Первое утверждение доказано.

Докажем второе утверждение. Пусть задана разбивающая раскраска графа $G^{0}$, $c\left(a_{1}\right)=-1, c\left(a_{2}\right)=+1$. Докажем, что тогда $c\left(b_{1}\right)=c\left(b_{2}\right)$. Предположим противное, пусть $c\left(b_{1}\right)=-t, c\left(b_{2}\right)=t, t \in\{-1,+1\}$. Так как цвета ребер $\left(a_{1}, B\right),\left(a_{2}, B\right)$ и $\left(b_{1}, B\right)$, инцидентных вершине $B$, равны соответственно $-1,+1$ и $+t$, то цвет оставшегося ребра $\left(d_{2}, B\right)$ равен $-t, c\left(d_{2}, B\right)=c\left(d_{2}\right)=-t$. Цвета ребер $\left(a_{1}, D\right),\left(b_{2}, D\right),\left(d_{2}, D\right)$, инцидентных вершине $D$, равны соответственно $-1,-t$ и $-t$; следовательно, $t=-1$, а цвет ребра $\left(c_{2}, D\right)$, инцидентного $D$, равен -1 .

Вершине $A$ инцидентны два ребра цвета +1 , ребра $\left(a_{2}, A\right),\left(b_{2}, A\right)$, следовательно, оставшиеся два ребра, инцидентные $A$, имеют цвет $-1, c\left(c_{1}, A\right)=c\left(d_{1}, A\right)=-1$; $c\left(c_{1}\right)=c\left(d_{1}\right)=-1$. Но тогда все четыре ребра, инцидентные вершине $C$, оказываются одного цвета, $c\left(b_{1}, C\right)=c\left(c_{1}, C\right)=c\left(c_{2}, C\right)=c\left(d_{1}, C\right)=-1$, что противоречит определению разбивающей раскраски. Таким образом, если $c\left(a_{1}\right) \neq c\left(a_{2}\right)$, то $c\left(b_{1}\right)=c\left(b_{2}\right)$. Лемма доказана.

Используем ее для доказательства нераскрашиваемости простого графа $G \in C_{3}^{0}$, изображенного на рис. 3. 


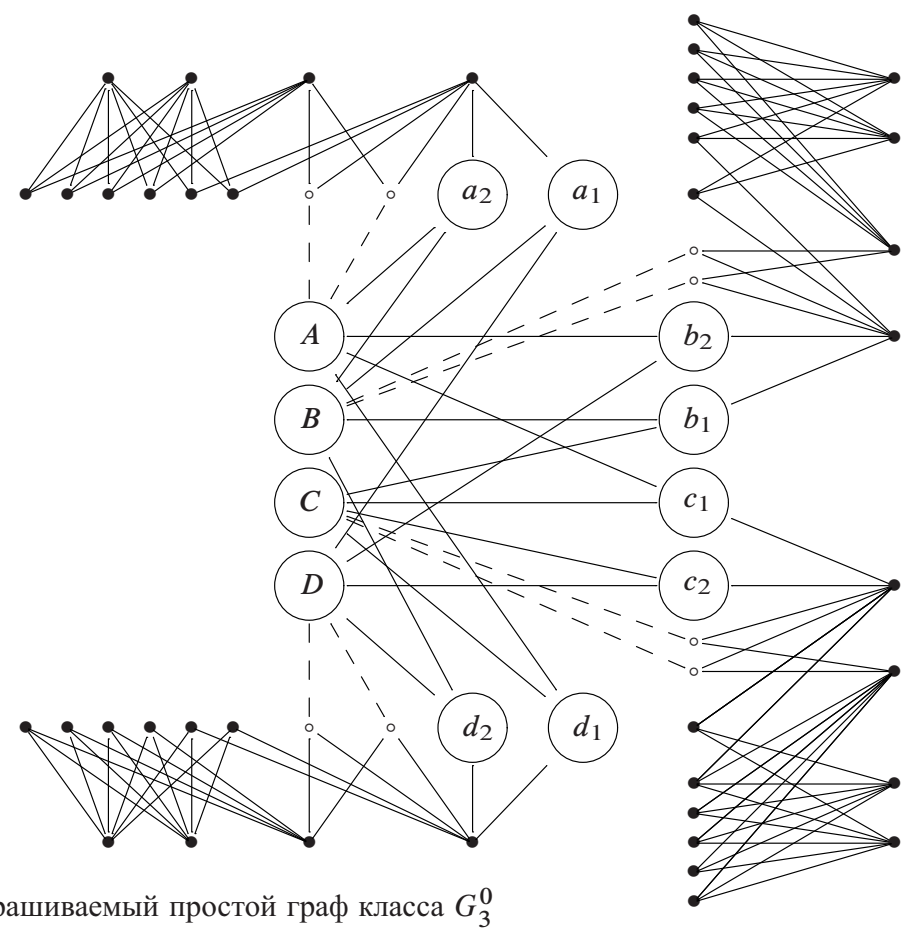

Рис. 3. Нераскрашиваемый простой граф класса $G_{3}^{0}$

Опишем структуру графа $G$. Выбраны четыре экземпляра $\left(G_{i}, 1 \leqslant i \leqslant 4\right)$ графа $G_{0}$, висячие вершины первого из них обозначены $a_{1}, a_{2}$, второго $-b_{1}, b_{2}$, третьего $-c_{1}$, $c_{2}$, четвертого $-d_{1}, d_{2}$. Затем добавлены четыре вершины $A, B, C, D$ : вершина $A$ соединена ребрами с вершинами $a_{1}$ и $a_{2}$, локальные степени которых в $G_{1}$ равны двум (в графе $G_{0}$ соответствующие вершины обозначены $y_{1}, y_{2}$ и разноцветны при любой разбивающей раскраске $G_{0}$ ), аналогичным образом проведены ребра от вершин $B, C$ и $D$ к соответствующим вершинам, локальные степени которых равны двум в $G_{2}, G_{3}$ и $G_{4}$ соответственно (на рисунке эти восемь ребер показаны пунктиром). В завершение построения графа проведены ребра $\left(A, a_{2}\right),\left(A, b_{2}\right),\left(A, c_{1}\right),\left(A, d_{1}\right) ;\left(B, a_{1}\right),\left(B, a_{2}\right),\left(B, b_{1}\right)$, $\left(B, d_{2}\right) ;\left(C, b_{1}\right),\left(C, c_{1}\right),\left(C, c_{2}\right),\left(C, d_{1}\right) ;\left(D, a_{1}\right),\left(D, b_{2}\right),\left(D, c_{2}\right),\left(D, d_{2}\right) ;$ соответствующий реберно-порожденный подграф изоморфен графу $G^{0}$ (рис. 2).

Предположим, что граф $G$ раскрашиваем и выбрана некоторая разбивающая раскраска. Индуцированная раскраска является разбивающей раскраской для каждого из подграфов $G_{i}$; поэтому, в соответствии с первым пунктом леммы, две вершины подграфа $G_{i}$, $1 \leqslant i \leqslant 4$, концевые для пунктирных ребер, имеют разные цвета. Отсюда следует, что индуцированная раскраска является разбивающей раскраской и для подграфа $G^{0}$, полученного удалением пунктирных ребер из реберно-порожденного подграфа (класса $\left.G_{3}\right)$ на множестве ребер, инцидентных вершинам $A, B, C, D$. Из первого пункта леммы, последовательно примененного к каждому из $G_{i}, 1 \leqslant i \leqslant 4$, следует, что $c\left(a_{1}\right) \neq c\left(a_{2}\right)$, $c\left(b_{1}\right) \neq c\left(b_{2}\right), c\left(c_{1}\right) \neq c\left(c_{2}\right), c\left(d_{1}\right) \neq c\left(d_{2}\right)$. Но это противоречит второму пункту леммы, согласно которому $c\left(a_{1}\right)=c\left(a_{2}\right)$ либо $c\left(b_{1}\right)=c\left(b_{2}\right)$. Следовательно, граф $G$ нераскрашиваем. В рассмотренном двудольном графе $G(V, W, E)$ пусть $V=\left\{v_{1}, \ldots, v_{n}\right\}$ и $W=\left\{w_{1}, \ldots, w_{L}\right\}-$ множества вершин степени 6 и 3 соответственно; $n=20, L=40$. Построим набор $W^{\prime}$ трехсимвольных слов $w_{i}^{\prime}, 1 \leqslant i \leqslant L$, над алфавитом $A=\{1, \ldots, n\}$, 
включая в $i$-е слово $w_{i}^{\prime}$ символ $j$, если $\left(w_{i}, v_{j}\right) \in E$; число вхождений каждого символа алфавита в слова набора $W^{\prime}$ равно шести. Из нераскрашиваемости графа $G$ вытекает следующее утверждение.

Предложение 1. Не существует разбиения $W^{\prime}$ на два поднабора, в слова каждого из которых любой символ алфавита А входит в точности три раза.

Реберное хроматическое число двудольного графа равно максимальной степени вершины [2], в данном случае - шести. Рассмотрим правильную реберную раскраску графа $G$ в шесть цветов, $1, \ldots, 6$, и разместим слова набора $W^{\prime}$ в $L \times 6$ матрице $M$ по правилу: если $j-$ символ слова $w_{i}^{\prime}$ и цвет ребра $\left(w_{i}, v_{j}\right)$ в правильной реберной раскраске равен $k$, то $M_{i k}=j$. В результате каждый символ алфавита представлен в любом столбце матрицы $M$ в точности один раз, а в каждой строке все три ненулевых элемента попарно различны.

Предложение 2. Дефрагментации матриць $M$ не существует.

Доказательство. В самом деле, пусть дефрагментация матрицы $M$ существует, и ее результат обозначен через $M^{\prime}$. Как в первом, так и в последнем столбцах матрицы $M^{\prime}$ каждый символ алфавита представлен в точности один раз. Последнее, ввиду сплошного размещения символов алфавита в строке, означает, что $n$ слов набора $W^{\prime}$ размещены в первых трех столбцах (поднабор $W_{1}^{\prime}$ ), а $n$ слов - в последних трех столбцах матрицы $M^{\prime}$ (поднабор $W_{2}^{\prime}$ ). Так как при этом в любом столбце $M^{\prime}$ каждый символ алфавита представлен в точности один раз, получено разбиение, противоречащее предложению 1. Предложение 2 доказано.

\section{Список литературы}

1. Гэри М., Джонсон Д., Вычислительные машины и труднорешаемые задачи. Мир, Москва, 1982.

2. Свами М., Тхуласираман К., Графы, сети и алгоритмыл. Мир, Москва, 1984.

Статья поступила 22.01.2008. 